\title{
One Stage Conversion of an Infected Fused Knee to Total Knee Replacement - A Surgical Challenge
}

\author{
Mukartihal Ravikumar, Daniel Kendoff*, Mustafa Citak, Stefan Luck, Thorsten Gehrke and Akos Zahar
}

Helios ENDO-Klinik Hamburg, Department of Orthopaedic Surgery, Holstenstraße 2, 22767 Hamburg, Germany

\begin{abstract}
Background and Purpose: Two-stage revision arthroplasty is a common technique for the treatment of infected total knee replacement. Few reports have addressed the conversion of a fused knee into a total knee replacement. However, there is no case reported of converting an infected fused knee into a hinge knee using a one-stage procedure.

Methods: We report on a 51-year old male patient with an infected fused knee after multiple surgeries.

Results and Interpretation: A one-stage conversion of septic fused knee into total knee arthroplasty by a rotational hinge prosthesis was performed. The case highlights that with profound preoperative assessment, meticulous surgical technique, combined antibiotic treatment and the right implant, one-stage revision in a surgical challenge may have a role as a treatment option with good functional outcome.
\end{abstract}

Keywords: Infection, knee arthrodesis, one-stage revision, total knee arthroplasty, two-stage revision.

\section{INTRODUCTION}

Conversion of a fused knee to a total knee arthroplasty (TKA) is a challenging procedure with many possible complications, including a high incidence of loosening, arthrofibrosis and infection. A high incidence of diverse complications (53 to 57\%) has been reported [1-3].

Due to the surgical challenges a constrained rotational hinge was established at the ENDO-Klinik in 1979 [4]. Recent studies have shown that the use of this type of knee implant results in good long term survivorship and these results have been attributed to improved surgical technique, adherence to strict preoperative selection criteria, and improvements in prosthetic design [5]. A recent study showed that a constrained rotational hinge knee has helped to overcome the instability and to convert the fused knee into a mobile joint [6]. Rotational hinge allows axial rotation and reduces the friction acting on the prosthesis.

We present a case of a one-stage septic revision from a fused knee to a rotational hinge knee prosthesis. As we are aware, this young and active patient is the only one who underwent this procedure ever. The good mid- term results of this patient suggest that one- stage revision even in difficult preoperative conditions can have a role as a treatment option in young, motivated patients who have had a long history of pain, instability and limited range of motion. Most importantly, the technical aspects resulting in the survivorship of this device, which is now realised as current best practice in single-stage knee revisions will be discussed.

\section{PATIENT AND CASE DESCRIPTION}

A 51-years old male patient with an external diagnosed periprosthetic infection of the left knee with verification of

*Address correspondence to this author at the Department of Orthopaedic Surgery, Endo Klinik Hamburg, Holstenstrasse 2, D-22767 Hamburg, Germany; Tel: +49 403197 0; Fax: +49 403197 1963;

E-mail: Daniel.kendoff@endo.de methicillin-resistant Staphylococcus aureus (MRSA) was admitted to the ENDO-Klinik, Hamburg. He was associated with multiple comorbidities in terms of depression, hypertension, myocardial infarction, renal insufficiency, obesity, diabetes mellitus type 2 and hypercholesterolemia.

Twelve-years before admission, a diagnostic arthroscopy for the left knee was performed at a regional hospital following with a high tibial osteotomy (HTO) due to medial unicompartmental arthritis of the left knee. The patient developed an infection with the need of revision surgery utilizing an external fixator. After treating the infection, a total knee replacement (TKR) was performed at a university hospital. Six months postoperatively, the patient complained of pain over the medial aspect of the knee above and below the prosthesis. Further analyses showed a nickel and bone cement allergy of this patient. So a one-stage revision arthroplasty was performed with a cementless TKA in a regional hospital. One year after this procedure, a periprosthetic infection was diagnosed resulting in a revision surgery with fusion of the left knee. However, the infection was still verifiable with Staphylococcus capitis and Staphylococcus ureolyticus with the necessity of further softtissue revisions. Despite several revision surgeries including long-time antibiotic therapy the patient had still a persistent infection. One year before admission to the authors' hospital, the patient developed a draining sinus on the medial aspect of the left knee joint. Bacterial cultures from knee aspiration showed a methicillin-resistant Staphylococus aureus (MRSA) infection.

\section{CLINICAL AND RADIOLOGICAL EXAMINATION ON ADMISSION}

The physical examination on admission revealed a draining sinus $(1 \mathrm{~cm})$ on the medial aspect of the left knee joint (Fig. 1). No neurological deficits were seen on admission. The performed radiographs of the left knee (anteroposterior and lateral view) showed a loosening of the femoral component with periostal reactions of the distal 
femur (Fig. 2). The tibial component showed no signs of loosening. The performed weight bearing long leg radiographs revealed a mechanical leg axis of $8^{\circ}$ varus. Joint aspiration was done which grown Staphylococcus hominis, Staphylococus capitis and Corynebacterium species. A routine nasal smear from the patient was found MRSA positive.

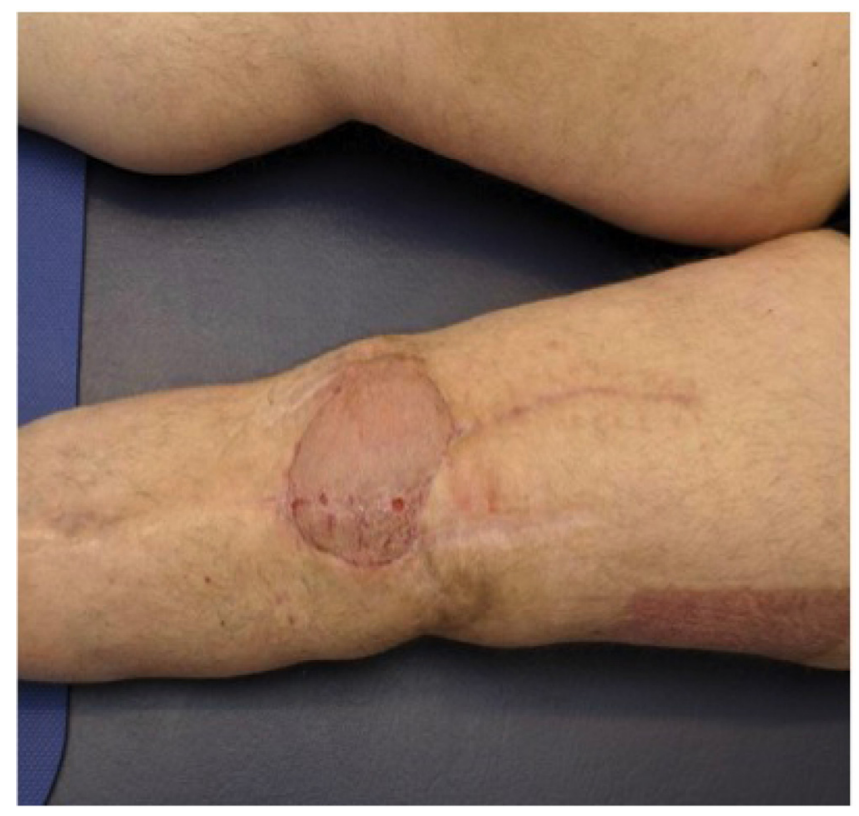

Fig. (1). Soft tissues are highly compromised by previous surgeries, draining sinus and plastic surgery covering.

\section{SURGICAL PROCEDURE}

Old scars in the line of the skin incision were excised. The existing sinus was integrated into the incision and was radically excised to the joint capsule. The procedure was started without tourniquet; consequently boundaries between infected tissue, scar and surrounding healthy bleeding soft tissue (and bone) were separated and precisely excised during the debridement. All non-bleeding tissues and related bony structures were removed. At the early stage of operation, during debridement, biopsy material (5 tissue samples) was taken from all relevant areas of the surgical site for combined microbiological and histological evaluation [7-9]. A full synovectomy, including the posterior aspects of the knee, was done. Consequent resection of all ligaments including the collaterals was done, to perform this radical and complete soft tissue resection. After taking the last microbiological sample, the systemic antibiotic therapy was started by administering a first dose of intravenous Vancomycin and Rifampicin through a central venous catheter.

Topical antibiotic treatment with revision PMMA bone cement was achieved by 2 grams of Vancomycin, 1 gram of Clindamycin and 1 gram of Gentamicin (calculated on 40 grams PMMA) based on the microbiological testing of preoperative aspiration samples.

For the removal of the implant high speed burrs, curved power saw blades and special extraction instruments were utilised. The explantation of the well fixed tibial component caused no considerable loss of bone stock (Fig. 3).

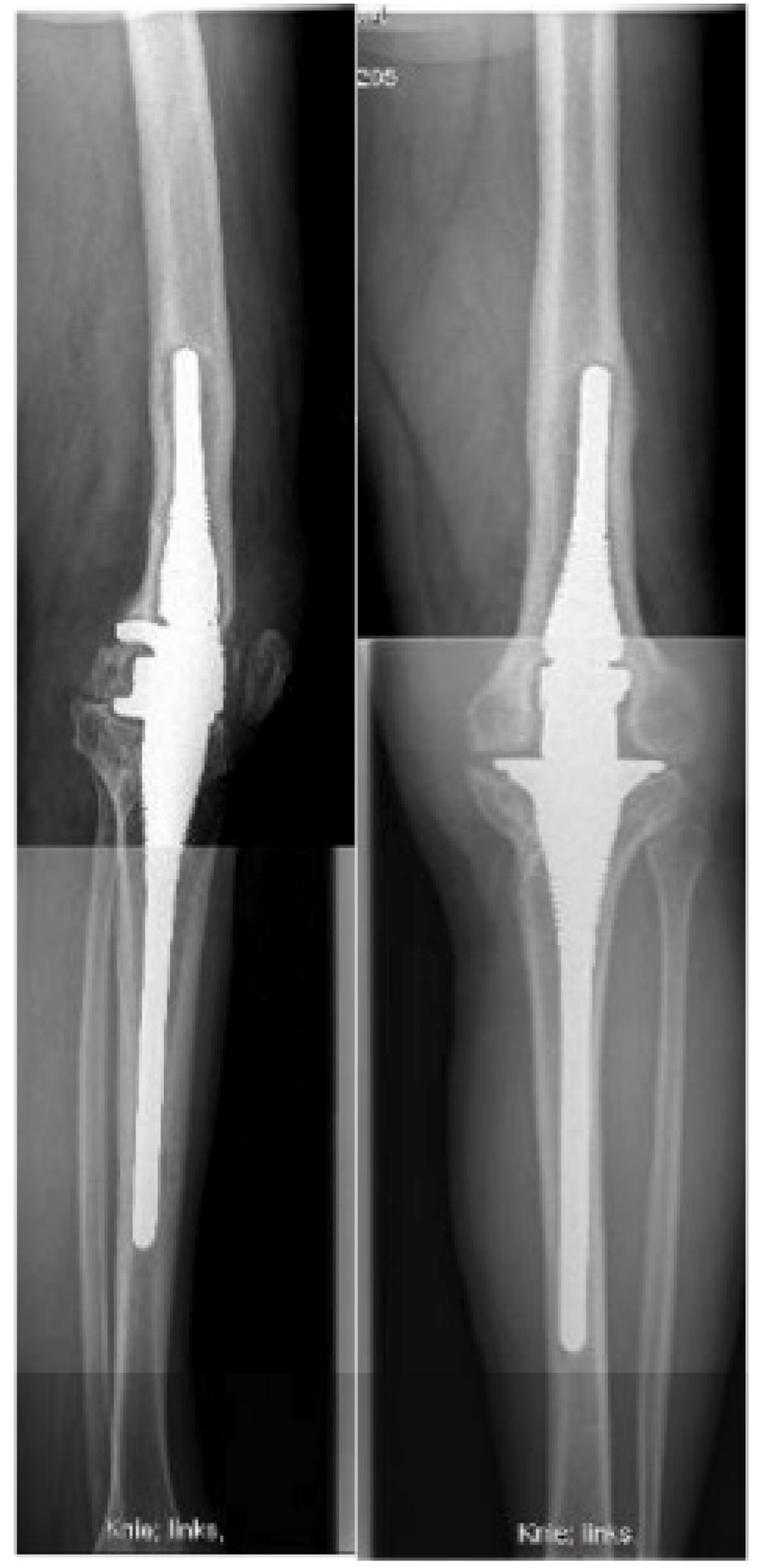

Fig. (2). Anteroposterior and lateral radiographs showing the fused knee with implants in situ.

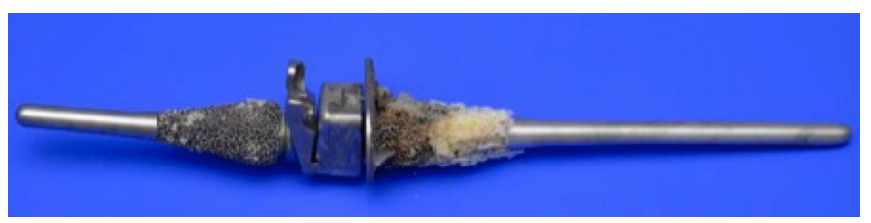

Fig. (3). Uncemented arthrodesis device after removal from left knee.

We used pulsatile jet-lavage with polymeric biguanidhydrochlorid (polyhexanid) throughout the whole procedure, after the microbiological samples were taken. After the removal of the infected implants and radical debridement the complete surgical team re-scrubbed, the patient was redraped and new instruments were used for re-implantation. 
A second dose of intravenous Vancomycin and Rifampicin was administered.

After thorough debridement as described and multiple lavages we implanted a rotational hinge knee prosthesis (W.LINK, Hamburg, Germany) with antibiotic loaded bone cement (BIOMET Refobacin Revision Bone Cement).

Cultures from intraoperative specimen showed growth of Staphylococcus capitis and B-haemolytic Streptococci sensitive to vancomycin and rifampicin. He was treated with this antibiotic combination and the wound was inspected after 48 hours.

Due to a wound healing disturbance the patient was transferred to a plastic surgery department to cover the wound with a gastrocnemius flap. The wound and the flap healed well. However, the intravenous antibiotic therapy was continued for another 12 days.

The patient came to our outpatient clinic for the three months follow-up and the wound was healed and he had flexion of 70 degrees and almost full extension and pain free range of motion. Additionally his C-reactive protein (CRP) and erythrocyte sedimentation rate (ESR) were within normal limits. He was followed up once again 6 months postoperatively and he achieved 90 degrees of flexion (Fig. 4) and pain free independent walking without crutches or any other support.

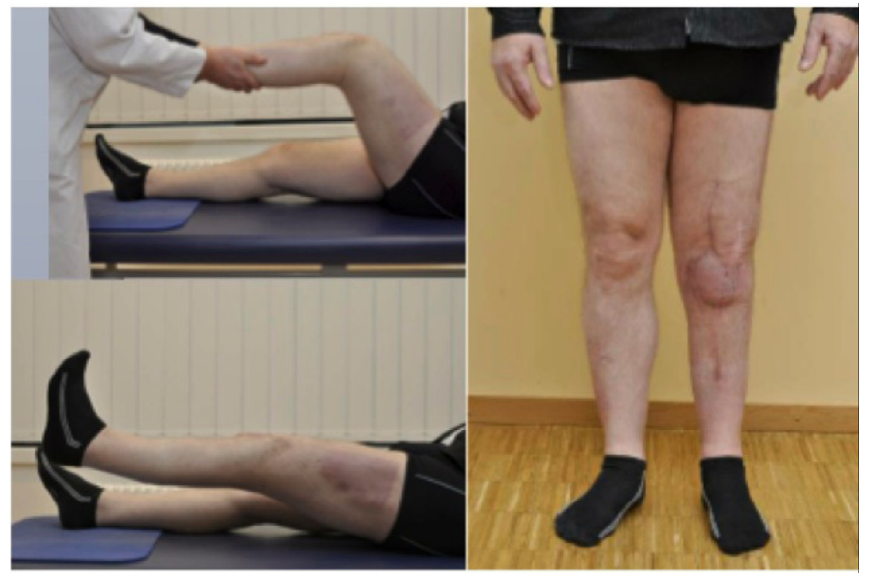

Fig. (4). Clinical images showing range of movements and limb axis.

At his most recent follow-up evaluation 2 years after surgery all clinical parameters (blood, $\mathrm{x}$-ray and clinical evaluation) (Fig. 5) showed no signs or evidence of infection. He has come back to normal routine life activities after 12 years of painful life.

\section{DISCUSSION}

The surgical success of a one staged approach does not only depend on the distinct and complete removal of all foreign material, including complete cement mantle and restrictors, furthermore the aggressive and complete debridement of any infected bone and soft tissue in combination only allows a high success rate [10].

The two-staged approach has become the method of choice for most surgeons world wide, with a reported reinfection incidence between $9 \%$ and $20 \%$. Although advocated as the gold standard, we established and followed a distinct one stage approach in our clinic since over 35 years in over $85 \%$ of all our infected TKA patients. Even though two stage is gold standard, Kubista et al. showed $15.8 \%$ patients had developed reinfection after the two-stage procedure [11].

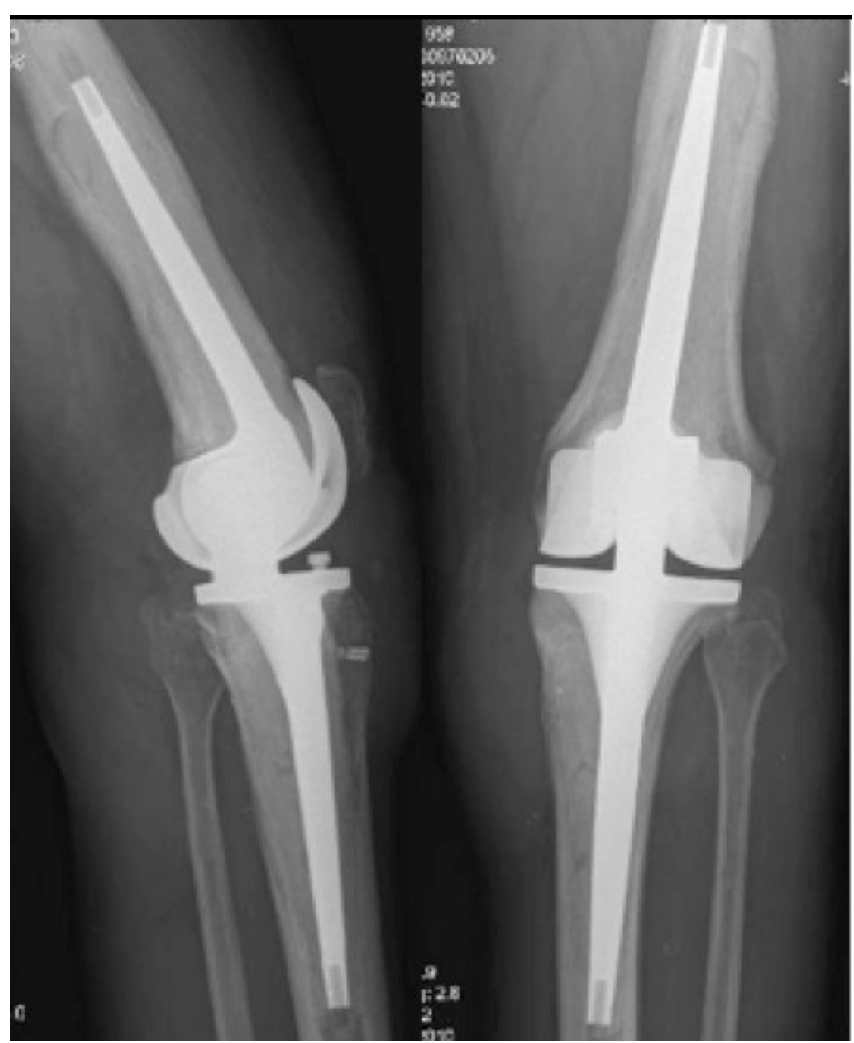

Fig. (5). Anteroposterior and lateral views two years after surgery showing components of hinged knee joint arthroplaty with no signs of loosening.

Accordingly far more studies have been published and emphasised about the two- or more-stage revision technique so far. Currently some very few studies evaluating the one stage exchange and its techniques are available [12-16]. Although most reports are from our own institution, some international experience using this technique exists, with comparable high success rates between $90 \%$ and $75 \%$, depending on the time of follow up.

Buechel was able to show in 22 patients treated with a one stage exchange and i.v. antibiotics for 4-6 weeks a $90 \%$ success rate for eradication of infection after 10 years, however only under a continuation of oral antibiotics for 612 months [13]. A systematic review by Silva confirmed a success rate of around $90 \%$, with the one staged approach, however no distinct information was provided regarding the respective length of follow-up in all described studies [17].

Single stage revision for infection is well established for total hip arthroplasty. Wrobleski first published his experience in 1986 and subsequently published his long term results in 1995 [18]. More recently Callaghan [19] and Rudelli [20] reported their long term results at 10 years $(8 \%$ recurrence) and 5 years (6\% recurrence) respectively. It is perhaps surprising that single stage revision is widely accepted in hip arthroplasty but to date it does not carry the same level of support in the knee. 
Single stage revision for infection in TKA involves removal of the implant followed by debridement and immediate reimplantation of a new prosthesis, in combination with antibiotic loaded cement. This technique appears to be gaining popularity. Initial reports from the ENDO-Klinik [10] in Germany reported a success rate of $73 \%$. Goksan and Freeman [21] published their results in 1992 and only had recurrence in one patient out of 18 . Buechel [13] in a series of 22 patients showed a $90.9 \%$ success rate at an average of 10 years.

Our technique of single stage revision consists of an interval between the implant removal with thorough debridement and reimplantation of only a few minutes, just allowing enough time to re-gown and re-drape to utilise a second set of sterile instruments. It is considered as very important to identify the infecting organism prior to the single stage revision. This was done by aspiration of knee and assessment of culture and susceptibility report. We mix additional antibiotic powder to the manufactured clindamycin/gentamicin-loaded PMMA revision cement. In this case vancomycin was added as part of the complex therapy.

One stage revision avoids most of the drawbacks of a two-stage approach to the knee. It avoids mostly the problems of stiffness and arthrofibrosis of the joint, which sometimes is a challenge at two-stage surgery. It is more cost effective by saving the patient from having to undergo a second major surgery. It decreases the surgical and anaesthesiological risks for complications since it's just one procedure. All these factors make single stage revision an attractive option not only for the surgeons but also for the patients.

Bauer [22] published a multicenter retrospective study comparing single and two-stage revisions for infection and concluded that there was no difference between the two techniques in eradicating the infection. They also observed that after two-stage procedures, the knee outcome was excellent in $33 \%$ of patients while $40 \%$ of the knees had an excellent outcome after one-stage revision.

More evaluation of the clinical results is needed to compare the outcomes of single stage versus the more traditional two-stage procedure. If the results of one stage revision are comparable, then there are advantages for patients with infected TKA.

Besides some obvious surgical benefits, by eliminating a second major operative procedure, further major advantage arises from the relevantly reduced duration of treatment with postoperative systemic antibiotics. This rarely prolongs more than 14 days in our set up. The rationale for this has also been evaluated in a study by Hoad-Reddick, where the authors concluded, that a prolonged course of antibiotics does not seem to alter the incidence of recurrent or persistent infection, even after a two staged revision [23].

Periprosthetic infection with MRSA is quite problematic. Because of the reduced therapeutic possibilities it is associated with a higher rate of recurrence than the unselected group of patients as a whole. Most of the authors recommend at one-stage exchange arthroplasty using a combination of vancomycin and revision PMMA bone cement with clindamycin and gentamycin. Intraoperative use of an antiseptic solution and systemic administration of rifampicin as a combination therapy, as recommended by Zimmerli is a further possibility [14].

The physiotherapeutic plan in any one staged approach can not be generalised. Based on the variety of osseous defects, soft tissue compromising, extent of infection and further patient specific circumstances, an individual patient plan is developed. Although compromises between necessary immobilisation due to structural damage and general attempts of early mobilisation have to be made, we recommend a mobilisation as early as possible. However in this particular case weight bearing and ambulation was delayed for 3 weeks for the soft tissue management.

Technically the presence of a positive culture, based on a joint aspiration, with a respective antibiogram is mandatory preoperatively for a successful one-staged approach. A cemented implant fixation using topic antibiotics is the treatment of choice for our recommended single staged procedure. The essential success of the surgical procedure is directly related to the experience and communication of a designated microbiologist or infectious disease specialist, who develops the patient specific treatment plan. This includes specifications of the systemic and topic antibiotic regime. Explantation of the infected implant is followed by an extensive local soft tissue and bone debridement. Consequently implantation of the new cemented implant with antibiotics loaded bone cement becomes possible. A defined systemic antibiotic therapy follows, whereas early mobilisation should be started as soon as possible. The key to success is based on the well defined intra-hospital infrastructure to fulfill the complete pathway of proper preoperative planning, aggressive surgical approach and postoperative specific patient care.

After digging through the literature many reports above were against a one stage approach in this case. We made the decision to treat this patient with a single stage septic exchange considering the multiple previous surgeries and the co-morbidities. Since the one stage septic exchange is daily practice at the ENDO-Klinik the decision in this case was made with ease. However no case is reported in revising MRSA active infected fused knee into a total knee replacement in one stage.

\section{CONCLUSIONS}

Meticulous planning, radical debridement, appropriate antibiotic therapy and strict follow up in this case significantly improved the life activities of this patient, and avoided all the side effects of a two-stage procedure.

\section{CONFLICT OF INTEREST}

The authors confirm that this article content has no conflict of interest.

\section{ACKNOWLEDGEMENTS}

In memory of our colleague Bernd Schwantes who treated this patient. He passed away in 2011. No benefits in any form have been received or will be received from a commercial party related directly or indirectly to the subject of this article. No funds were received in support of this study. 


\section{REFERENCES}

[1] Cameron $\mathrm{HU}, \mathrm{Hu} \mathrm{C}$. Results of total knee arthroplasty following takedown of formal knee fusion. J Arthroplasty 1996; 11: 732-7.

[2] Kim YH, Kim JS, Cho SH. Total knee arthroplasty after spontaneous osseous ankylosis and takedown of formal knee fusion. J Arthroplasty 2000; 15: 453-60.

[3] Naranja RJ, Jr., Lotke PA, Pagnano MW, Hanssen AD. Total knee arthroplasty in a previously ankylosed or arthrodesed knee. Clin Orthop Relat Res 1996; 331: 234-7.

[4] Engelbrecht E, Nieder E, Strickle E, Keller A. Intracondylar knee joint prosthesis with rotation capacity- Endo model. Chirurg 1981; 52: 368-75.

[5] Petrou G, Petrou H, Tilkeridis C, et al. Medium-term results with a primary cemented rotating-hinge total knee replacement. A 7- to 15-year follow-up. J Bone Joint Surg Br 2004; 86: 813-7.

[6] Hartford JM, Goodman SB, Schurman DJ, Knoblick G. Complex primary and revision total knee arthroplasty using the condylar constrained prosthesis: an average 5-year follow-up. J Arthroplasty 1998; 13: 380-7.

[7] Atkins BL, Bowler IC. The diagnosis of large joint sepsis. J Hosp Infect 1998; 40: 263-74.

[8] Fink B, Makowiak C, Fuerst M, Berger I, Schafer P, Frommelt L. The value of synovial biopsy, joint aspiration and $\mathrm{C}$-reactive protein in the diagnosis of late peri-prosthetic infection of total knee replacements. J Bone Joint Surg Br 2008; 90: 874-8.

[9] Spangehl MJ, Masri BA, O'Connell JX, Duncan CP. Prospective analysis of preoperative and intraoperative investigations for the diagnosis of infection at the sites of two hundred and two revision total hip arthroplasties. J Bone Joint Surg Am 1999; 81: 672-83.

[10] von Foerster G, Kluber D, Kabler U. Mid- to long-term results after treatment of 118 cases of periprosthetic infections after knee joint replacement using one-stage exchange surgery. Orthopade 1991; 20: 244-52.

[11] Kubista B, Hartzler RU, Wood CM, Osmon DR, Hanssen AD, Lewallen DG. Reinfection after two-stage revision for periprosthetic infection of total knee arthroplasty. Int Orthop 2012; 36: $65-71$.
[12] Buechel FF. The infected total knee arthroplasty: just when you thought it was over. J Arthroplasty 2004; 19: 51-5.

[13] Buechel FF, Femino FP, D'Alessio J. Primary exchange revision arthroplasty for infected total knee replacement: a long-term study. Am J Orthop (Belle Mead NJ) 2004; 33: 190-8.

[14] Kordelle J, Frommelt L, Kluber D, Seemann K. Results of onestage endoprosthesis revision in periprosthetic infection cause by methicillin-resistant Staphylococcus aureus. Z Orthop Ihre Grenzgeb 2000; 138: 240-4.

[15] Siegel A, Frommelt L, Runde W. Therapy of bacterial knee joint infection by radical synovectomy and implantation of a cemented stabilized knee joint endoprosthesis. Chirurg 2000; 71: 1385-91.

[16] Siegel A, Frommelt L, Runde W, Engelbrecht E. Primary arthroplasty of infected hips and knees in special cases using antibiotic-loaded bone-cement for fixation. J Arthroplasty 2001; 16: 145-9.

[17] Silva M, Tharani R, Schmalzried TP. Results of direct exchange or debridement of the infected total knee arthroplasty. Clin Orthop Relat Res 2002; 404: 125-31.

[18] Wroblewski BM. One-stage revision of infected cemented total hip arthroplasty. Clin Orthop Relat Res 1986; 211: 103-7.

[19] Callaghan JJ, Katz RP, Johnston RC. One-stage revision surgery of the infected hip. A minimum 10-year followup study. Clin Orthop Relat Res 1999; 369: 139-43.

[20] Rudelli S, Uip D, Honda E, Lima AL. One-stage revision of infected total hip arthroplasty with bone graft. J Arthroplasty 2008; 23: 1165-77.

[21] Goksan SB, Freeman MA. One-stage reimplantation for infected total knee arthroplasty. J Bone Joint Surg Br 1992; 74: 78-82.

[22] Bauer T, Piriou P, Lhotellier L, Leclerc P, Mamoudy P, LortatJacob A. Results of reimplantation for infected total knee arthroplasty: 107 cases. Rev Chir Orthop Reparatrice Appar Mot 2006; 92: 692-700.

[23] Hoad-Reddick DA, Evans CR, Norman P, Stockley I. Is there a role for extended antibiotic therapy in a two-stage revision of the infected knee arthroplasty? J Bone Joint Surg Br 2005; 87: 171-4. 